\title{
Diferença entre volume de fluidos cristaloides intravenosos prescritos e infundidos em pacientes no pós-operatório precoce
}

\author{
Difference between intravenous crystalloid fluids prescribed and infused in \\ patients during early postoperative period
}

\author{
José Eduardo de Aguilar-Nascimento, TCBC-MT ; Breno Nadaf Diniz ${ }^{2}$; José de Souza Neves, ACBC-MT³
}

R E S U M O

\begin{abstract}
Objetivo: O objetivo deste trabalho foi auditar a real quantidade de fluídos cristalóides infundidos por via intravenosa em pacientes submetidos a operações abdominais de grande porte num hospital universitário. Métodos: Computou-se a carga hídrica total (CHT) de fluidos cristalóides intravenosos infundida diariamente do $1^{\circ}$ ao $4^{\circ}$ dia de $\mathrm{PO}$ em 31 pacientes submetidos à operações de grande porte. Comparou-se a CHT com a carga hídrica prescrita (CHP) pelo médico. A CHT foi definida como a somatória da CHP acrescida de diluentes e medicações intravenosas. O protocolo do serviço recomendava a hidratação venosa no peri-operatório entre 30 e 50 $\mathrm{mL} / \mathrm{Kg} /$ dia em pacientes com prescrição de jejum oral. A comparação entre CHT e CHP foi realizada em todos os dias de pósoperatório pelo teste $\mathrm{t}$ pareado. Estabeleceu-se em $5 \%$ o nível de significância estatística. Resultados: A CHT infundida do $1^{\circ}$ ao $4^{\circ}$ dia de pós-operatório foi de 12,8 (6,4-17,5) L. Desse total, 9,5 litros $(74,3 \%)$ corresponderam a CHP e 3,3 L $(25,7 \%)$ a diluentes e medicações venosas. Em todos os dias de pós-operatório a CHT foi significativamente maior que a $\mathrm{CHP}(\mathrm{p}<0.001)$. Até $\circ 3^{\circ}$ dia de PO os pacientes receberam uma CHT superior a $50 \mathrm{~mL} / \mathrm{kg} / \mathrm{dia}$. Conclusão: Conclui-se que a prescrição médica não contém o real volume de fluidos cristalóides intravenosos infundido. O volume de diluentes e medicações intravenosas pode chegar a $25 \%$ da carga hídrica prescrita.
\end{abstract}

Descritores: Infusões intravenosas. Assistência Perioperatória; Hidratação; Cuidados Pós-Operatórios.

\section{INTRODUÇÃO}

A terapia de hidratação perioperatória é de vital importância no controle do paciente cirúrgico. No entanto, a reposição exagerada de fluidos intravenosos no perioperatório está associada a uma série de complicações'. Nesse contexto, protocolos multimodais tais como o ERAS e o ACERTO, que incluem a restrição de fluidos intravenosos como um de seus pilares, têm demonstrado que podem acelerar a recuperação pós-operatória ${ }^{2,3}$. Uma forte evidência para se restringir fluidos intravenosos foi mostrada com um elegante estudo randomizado multicentrico na Europa que mostrou a importância de um protocolo de restrição hídrica na diminuição da morbidade pós-operatória quando comparado a protocolo convencional de infusão intravenosa ${ }^{4}$. Depreende-se da literatura atual que existe uma relação clara entre excesso de volume administrado por via intravenosa e aumento de peso, maior tempo de internação, complicações pulmonares e sépticas ${ }^{1-6}$.

Na rotina de hidratação venosa peri-operatória é mister o cálculo aproximado do volume total a ser infundido diariamente nos pacientes. Em pacientes submetidos a operações de grande porte e gravemente enfermos essa atenção é especialmente importante, pois, o volume infundido é alto. A hipótese deste trabalho é que nem sempre esse volume é bem monitorado. Assim, diluentes e medicações liquidas para uso intravenoso muitas vezes não são computadas no cálculo do total de fluidos infundidos. Podese especular então que, se devidamente monitorado, o volume de fluidos infundidos por via intravenosa pode ser bem maior que o imaginado pelo cirurgião.

O objetivo primário deste trabalho foi auditar a real quantidade de líquidos infundidos nos pacientes cirúrgicos do Hospital Universitário Júlio Muller confrontando estes valores com os que constavam na prescrição médica.

\section{MÉTODOS}

Foram incluídos no estudo 31 pacientes (22 (71\%) do sexo masculino e nove (29\%) do feminino) com média de idade de 56 (16-81) anos e peso médio de $58(32-88) \mathrm{kg}$ submetidos à cirurgia de grande porte (tabela1), no Hospital Universitário Julio Muller no período de janeiro a dezembro de 2004. As características demográficas e clínicas dos pacientes operados encontram-se na tabela 2. Nesse

Trabalho realizado no Departamento de Clínica Cirúrgica da Faculdade de Ciências Médicas da Universidade Federal de Mato Grosso - Hospital Universitário Júlio Muller - Cuiabá - MT - BR.

1. Professor Titular do Departamento de Cirurgia da Faculdade de Medicina da Universidade Federal de Mato Grosso - Cuiabá - MT - BR. 2. Aluno do $6^{\circ}$ ano de Medicina da Faculdade de Medicina da Universidade Federal de Mato Grosso - Cuiabá - MT - BR. Bolsista PIBIC-VIC/CNPa. 3. Professor Assistente do Departamento de Cirurgia da Faculdade de Medicina da Universidade Federal de Mato Grosso - Cuiabá - MT - BR. 
período, o protocolo do serviço recomendava a hidratação venosa no peri-operatório entre 30 e $50 \mathrm{~mL} / \mathrm{Kg} / \mathrm{dia}$ em pacientes com prescrição de jejum oral. Foram excluídos pacientes que receberam nutrição parenteral, sangue e hemoderivados nesse período.

De maneira retrospectiva, registrou-se todo o volume infundido no período intra-operatório e em todos os dias de pós-operatório até o quarto dia. Contabilizou-se todo o volume utilizado para diluir medicações, volume das próprias medicações e diversos tipos de soros cristalóides realizados (solução salina à 0,9\%, Ringer, Ringer lactato e soro glicosado à 5 e 10\%). O volume de diluentes utilizado de rotina pelo serviço de enfermagem para os medicamentos mais utilizados pode ser visto na tabela 3. Denominouse de carga hídrica total (CHT) a soma do total desse volu-

Tabela 1 - Número e percentual das 31 operações realizadas.

\begin{tabular}{lrc}
\hline CIRURGIAS & N & $\%$ \\
\hline Gastrectomias & 14 & 50 \\
Colectomias & 9 & 32 \\
Duodenopancreatectomias & 5 & 18 \\
\hline
\end{tabular}

me. Pela prescrição médica somou-se o total de fluidos prescritos em forma de solução salina prescrito pelo médiCO, carga hídrica prescrita (CHP).

A comparação entre CHT e CHP foi realizada em todos os dias de pós-operatório pelo teste t pareado. Estabeleceu-se em $5 \%$ o nível de significância estatística.

\section{RESULTADOS}

A CHT infundida do $1^{\circ}$ até o $4^{\circ}$ dia de pós-operatório foi em média 12,8 [6,4-17,5] litros. Desse total, 9,5 litros $(74,3 \%)$ corresponderam a fluidos cristalinos prescritos (CHP) e 3,3 litros $(25,7 \%)$ a diluentes e medicações venosas. Isso significa que aproximadamente $1 / 4$ da CHT recebida foi composta por diluentes e medicações intravenosas.

O volume prescrito (CHP) e o realmente infundido $(\mathrm{CHT})$, individualizados do $1^{\circ} \mathrm{ao} 4^{\circ} \mathrm{PO}$, são mostrados na figura 1 e 2 na forma de $\mathrm{mL} / \mathrm{Kg} / \mathrm{dia}$ e $\mathrm{mL} / \mathrm{dia}$, respectivamente. Em todos os dias de pós-operatório a CHT foi significativamente maior que a $\mathrm{CHP}(\mathrm{p}<0.01)$. Até o $3^{\circ}$ dia de $\mathrm{PO}$ os pacientes receberam uma CHT superior a $50 \mathrm{~mL}$ $\mathrm{kg} / \mathrm{dia}$.

Tabela 2 - Características demográficas e clínicas dos 31 pacientes.

\begin{tabular}{lrc}
\hline Variável & \multicolumn{2}{c}{ Valor } \\
\hline Sexo (M/F) & $21 / 9$ & \\
Idade (mediana e variação) & 56 & $(16-81)$ \\
Albumina (mediana e variação) & 3,4 & $(2,4-4,1)$ \\
Escore ASA (I/II/III) & $7 / 17 / 7$ & \\
Tipo de Anestesia (n, \%) & & $(74,2)$ \\
$\quad$ Geral & 23 & $(25,8)$ \\
$\quad$ Geral + Bloqueio & 8 & $(5-89)$ \\
Tempo de operação (minutos) (mediana e variação) & $270(160-510)$ \\
Tempo de internação (dias) (mediana e variação) & 12 & $(52,5)$ \\
Morbidade pós-operatória (n, \%) & 10 & $(9,7)$ \\
Mortalidade (n, \%) & 3 & $(9,7)$ \\
\hline
\end{tabular}

Tabela 3 - Volume de diluição com água destilada e volume final das medicações utilizadas com mais frequência.

\begin{tabular}{lccr}
\hline Medicamento & Apresentação $(\mathrm{mL})$ & Diluente $(\mathrm{mL})$ & Volume final (mL) \\
\hline Cefalotina & Liofílica & 100 & 100 \\
Amicacina & 2 & 100 & 100 \\
Metronidazol & 150 & - & 150 \\
Vancomicina & Liofílica & 20 & 20 \\
Ceftozidima & Liofilica & 100 & 100 \\
Dipirona & 2 & 20 & 22 \\
Metoclopramida & 2 & 20 & 22 \\
Cimetidina & 2 & 20 & 22 \\
Escopolamina & 5 & 20 & 25 \\
Tramadol & 2 & 100 & 102 \\
Furosemida & 2 & 20 & 22 \\
\hline
\end{tabular}




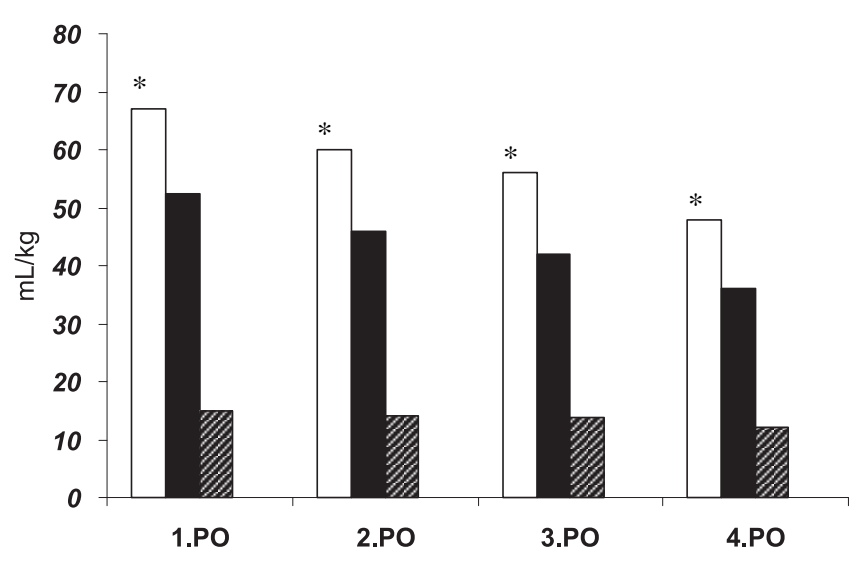

Figura 1 - Volume de líquido infundido $(\mathrm{m} / \mathrm{Kg})$ do primeiro ao quarto dia de pós-operatório. Coluna branca = carga hídrica total $(\mathrm{CHT})$; coluna preta = carga hídrica prescrita $(C H P)$, coluna riscada $=$ volume de medicamentos e diluentes. ${ }^{*} ; p<0.001$ vs. CHP em cada dia de pós-operatório $(P O)$

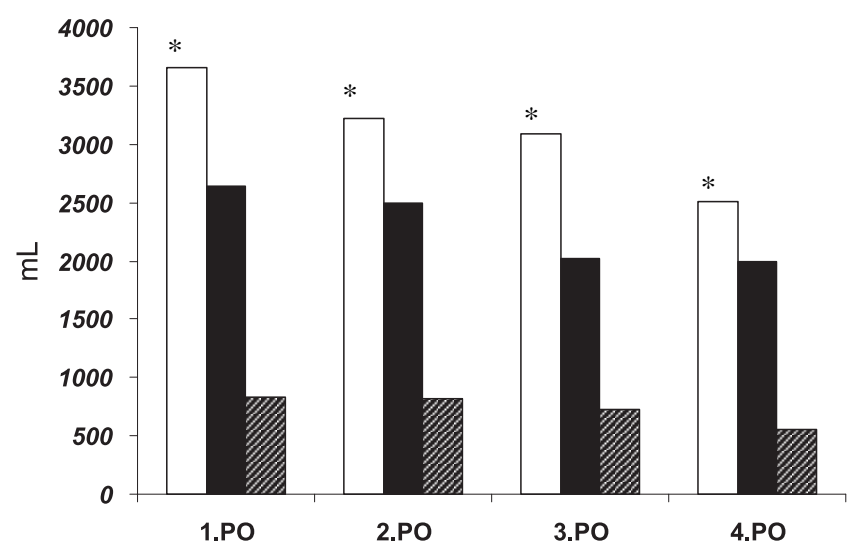

Figura 2 - Carga hídrica total (CHT) e prescrita (CHP) de cristalóides por via intravenosa nos 31 pacientes até o $4^{\circ}$ dia de pós-operatório. Coluna branca = carga hídrica total $(\mathrm{CHT})$; coluna preta = carga hídrica prescrita $(C H P)$, coluna riscada $=$ volume de medicamentos $e$ diluentes. *; $p<0.001$ vs. CHP em cada dia de pósoperatório $(P O)$.

\section{DISCUSSÃO}

O volume hídrico ideal para a reposição líquida no pós-operatório e a escolha do tipo de solução permanece sob debate. Há poucos trabalhos sobre o assunto e o debate entre reposição restrita ou liberal é atual ${ }^{7}$. As reposições liberais, tradicionalmente utilizadas, são atualmente vistas com reserva. Vários autores têm demonstrado a incidência maior de deiscência de suturas, fístulas e prolongamento do tempo de internação no pós-operatório em que os pacientes tiveram uma carga hídrica mais generosa ${ }^{4,6}$. Sabe-se que as soluções cristalóides, por exemplo, migram com certa celeridade para o espaço extracelular, ocasionando edema, diminuindo a tensão de oxigênio tecidual, com previsíveis conseqüências negativas no que se refere ao processo cicatricial. O retardo no esvaziamento gástrico, prolongamento de íleo pós-operatório, retardo na eli- minação de flatos e fezes são também observadas em pacientes com hidratação mais liberal ${ }^{6}$. Na esfera cardiológica e pulmonar o excesso de volume pode determinar hipertensão, cardiopatias e edema com hipoxemia. Em contrapartida, reposições volêmicas mais econômicas podem, se insuficientes, estar relacionadas com complicações tais como insuficiência renal aguda e déficits na perfusão tecidual com repercussões negativas, como por exemplo, na cicatrização ${ }^{7}$.

Este estudo colheu dados do ano de 2004, anterior ao novo protocolo ACERTO implantado no Serviço em $2005^{5}$. Após a implementação do protocolo ACERTO na enfermaria de Clínica Cirúrgica do Hospital Universitário Júlio Muller impondo restrição hídrica intravenosa no perioperatório houve significativa queda do tempo de internação e complicações pós-operatórias, quando comparou-se ao período pré-ACERTO ${ }^{5 ; 8}$. Estes resultados são semelhantes a estudos clínicos recentes da literatura ${ }^{9-11}$. O tipo de fluido intravenoso parece ser muito importante e o uso de colóides pode diminuir significativamente a chance de sobrecarga hídrica $^{12}$. Sobrecarga hídrica principalmente as custas de fluidos cristalóides deve ser evitada especialmente em ido$\operatorname{sos}^{13}$.

Neste trabalho observou-se que o volume prescrito pelo médico era ultrapassado em 25 a 30\% pelo total de diluentes e medicações venosas. Isso significou uma infusão de $50 \mathrm{~mL} / \mathrm{Kg} /$ dia até o $3^{\circ}$ dia de pós-operatório. Este resultado é extremamente significativo e permite afirmar que há uma hidratação bastante superior ao que o médico recomendou ao paciente. O volume infundido além da prescrição pode chegar a quase $1 \mathrm{~L}$ ao dia.

Tendo-se em vista que hidratações liberais associam-se com mais tempo de internação e complicações ${ }^{1-8}$, cirurgiões e anestesistas devem atentar a prática diária da enfermagem em diluir medicações. Esta atenção ao volume total da prescrição nem sempre é seguida. Uma recente investigação em hospital inglês demonstrou claramente que médicos não atentam a esse vital problema na hora de prescrever $^{14}$.

O conjunto dos dados apresentados neste trabalho mostra que é imperativo somar o volume da diluição e toda medicação injetável para obter-se a real carga hídrica injetada. Esse percentual diário de fluidos cristalóides não mensurados pode, a princípio, parecer pequeno, porém, certamente faz grande diferença quando é plenamente calculado. Ganho de peso, aumento de morbidade e mortalidade pós-operatória correlacionamse significantemente com a CHT ${ }^{4,15}$. Na nossa experiência quando comparamos dois protocolos de infusão cristalóide em cirurgias de grande porte ocorreu um maior numero de complicações, principalmente as pulmonares, e um aumento do tempo de internação no grupo que recebeu um aporte hídrico tradicional em comparação a um protocolo restritivo ${ }^{16}$.

Este estudo mostrou que o volume de hidratação final pode aumentar em $25 \%$ se for computado o volume de diluentes e de medicações injetáveis. Conclui-se então que a prescrição médica não contém o real volume de 
cristalóides infundido por via intravenosa e, por conseguinte, deve-se estar atento para, ao contabilizar o balanço hídrico, incluir os fluidos infundidos em conjunto com medicações venosas.

\section{A B S T R A C T}

Objective: The aim of this study was to audit the real amount of crystalloid intravenous fluids infused in patients underwent major abdominal operations in a University hospital. Methods: The whole intravenous crystalloid fluid load (CHT) infused from the $1^{\text {st }}$ to the $4^{\text {th }}$ postoperative day in 31 patients underwent major abdominal operations was registered. This amount was compared to the volume daily prescribed (CHP) by the physician. CHT was defined as the sum of CHP plus diluents and intravenous drugs received by the patients. Hydration protocol of the service was 30-50 $\mathrm{mL} / \mathrm{Kd} /$ day for patient with nil per os prescription. Comparisons between CHT and CHP was done in each postoperative day using paired T test. A 5\% level was established as significant.Results: CHT summed from $1^{\text {st }}$ to $4^{\text {th }} P O$ days was (mean and range) 12.8 (6.4-17.5) L corresponding to $9.5 \mathrm{~L}(74.3 \%)$ of CHP and $3.3 \mathrm{~L}(25.7 \%)$ of diluents and intravenous drugs. In each postoperative day, CHT was significantly greater than CHP $(p<0.001)$. Until the 3rd PO day patients received a CHT greater than $50 \mathrm{~mL} / \mathrm{Kg} /$ day. Conclusion: Medical prescription does not contain the real amount of crystalloid intravenous fluids infused. Diluents and intravenous drug may reach $25 \%$ of the intravenous fluids load.

Key words: Intravenous infusions. Perioperative care. Fluid therapy. Postoperative care.

\section{REFERÊNCIAS}

1. Joshi GP. Intra-operative fluid restriction improves outcomes after major elective gastrointestinal surgery. Anesth Analg 2005, 101 (2):601-5.

2. Aguilar-Nascimento JE, Salomão AB, Caporossi C, Silva RM, Cardoso EA, Santos TP. Acerto pós-operatório: avaliação dos resultados da implantação de um protocolo multidisciplinar de cuidados perioperatórios em cirurgia geral. Rev Col Bras Cir, 2006, (33):181-8.

3. Fearon $K C$, Ljungqvist $O$, von Meyenfeldt $M$, Revhaug A, Dejong $\mathrm{CH}$, Lassen $\mathrm{K}$, et al. Enhanced recovery after surgery. a consensus review of clinical care for patients undergoing colonic resection. Clin Nutr. 2005; 24(3):466-77.

4. Brandstrup B, Tonnesen H, Beier-Holgersen R, Hjortso E, Ording $H$, Lindorff-Larsen $\mathrm{K}$, et al. Effects of intravenous fluid restriction on postoperative complications: comparison of two perioperative fluid regimens: a randomized assessor-blinded multicenter trial. Ann Surg. 2003, 238 (5):641-8.

5. Aguilar-Nascimento JE, Bicudo-Salomão A, Caporossi C, Silva RM, Cardoso EA, Santos TP. Volume de fluído intravenoso e alta hospitalar precoce em colecistectomia aberta Rev Col Bras Cir. 2007, 34 (6):381-4.

5. Holte $\mathrm{K}$, Foss NB, Andersen J, Valentiner L, Liberal or restrictive fluid administration in fast-track colonic surgery: a randomized, double-blind study $\mathrm{Br} J$ Anaesth, 2007; 99:500-8.

6. Lobo DN, Bostock KA, Neal KR, Perkins AC, Rowlands BJ, Allison SP. Effect of salt and water balance on recovery of gastrointestinal function after elective colonic resection: a randomized controlled trial. Lancet. 2002, 359 (9320):1812-8.

7. Boldt J. Fluid management of patients undergoing abdominal surgery - more questions than answers; Eur J Anaesth; 2006; 23: 631-40.

8. Aguilar-Nascimento JE; Salomão AB; Caporossi C; Silva R; Santos TP; Cardoso EA. Enhancing surgical recovery in Central-West Brazil: The ACERTO protocol results. e-SPEN The European e-Journal of Clinical Nutrition and Metabolism. 2008; 3 (2): e78-e83. doi:10.1016/ j.eclnm.2008.01.003.

9. Holte K, Sharrock NE, Kehiet H. Pathophysiology and clinical implications of perioperative fluid excess. Br J Anaesth, 2002; 89: 622-30.
10. Macafee DA, Alisson SP, Lobo DN. Some interaction between gastrointestinal function and fluid and electrolyte homeostasis. Curr Opin Clin Nutr Metab Care 2005, 8(2):197-203.

11. Nisanevich V, Felsenstein I, Almogy G, Weissman C, Einav S, Matot I.; Effect of intraoperative fluid management on outcome after intraabdominal surgery. Anesthesiology. 2005,103(1):25-32.

12. Kvalheim VL, Rynning SE, Farstad M, Haugen O, Nygreen E, Mongstad A, et al. Fluid overload during cardiopulmonary bypass is effectively reduced by a continuous infusion of hypertonic saline/ dextran (HSD). Scand Cardiovasc J. 2008; 42(1):63-70.

13. Ferenczi E, Datta SS, Chopada A. Intravenous fluid administration in elderly patients at a London hospital: a two-part audit encompassing ward-based fluid monitoring and prescribing practice by doctors. Int J Surg. 2007;5(6):408-12.

14. Walsh SR, Cook EJ, Bentley R, Farooq N, Gardner-Thorpe J, Tang $T$, Gaunt ME, Coveney EC. Perioperative fluid management: prospective audit. Int J Clin Pract. 2008; 62(3):492-7.

15. Brandstrup B. Fluid therapy for the surgical patient. Best Pract Res Clin Anaesthesiol. 2006; 20(2):265-83.

16. de Aguilar-Nascimento JE, Diniz BN, do Carmo AV, Silveira EA, Silva RM. Clinical benefits after the implementation of a protocol of restricted perioperative intravenous crystalloid fluids in major abdominal operations. World J Surg. 2009; 33(5):925-30.

Recebido em 05/01/2009

Aceito para publicação em 05/03/2009

Conflito de interesse: nenhum

Fonte de financiamento: nenhuma

\section{Como citar esse artigo:}

Aguilar-Nascimento JE, Diniz BN, Neves JS. Diferença entre volume de fluidos cristalóides intravenosos prescritos e infundidos em pacientes no pós-operatório precoce. Rev Col Bras Cir. [periódico na Internet] 2010; 37(1). Disponível em URL: http://www.scielo.br/rcbc

\section{Endereço para correspondência:}

José Eduardo de Aguilar-Nascimento

E-mail: aguilar@terra.com.br 